\title{
CAMPOS DE TEMPOS DE TRÂNSITO OBTIDOS POR TRAÇAMENTO DE RAIOS SÍSMICOS EM CAMPOS DE VELOCIDADES COM PARAMETRIZAÇÃO POLINOMIAL
}

André Eduardo Calazans Matos de Souza, UFBA.

Dr.Wilson Mouzer Figueiró, UFBA

\section{Resumo}

Neste trabalho são obtidos campos de raios sísmicos e campos de tempos de trânsito para modelos de campos bidimensionais e isotrópicos de velocidades sísmicas de ondas compressionais parametrizados por funções polinomiais de duas variáveis. Tais funções são apresentadas com graus de liberdade crescentes visando representar modelos cada vez mais complexos. Em outras palavras: novos termos que são adicionados aos polinômios aumentam seus graus e, consequentemente, suas capacidades para representar modelos geológicos cada vez mais complicados. Aproximações para os vetores posição e vagarosidade com os dois primeiros termos de suas expansões em séries de Taylor, permitem o traçamento de raios sísmicos visando obter o máximo de cobertura do modelo. Tal cobertura é determinada por um espaçamento angular regular na direção de partida do raio na fonte. Tempos de trânsito são calculados ao longo dos raios traçados e estendidos para todo o modelo através de técnicas interpoladoras. Neste trabalho, foram consideradas cinco formas polinomiais para representação de campos de velocidades. As três primeiras formas foram usadas para representar campos genéricos de velocidades; a quarta representou uma dessas formas genéricas e, também, ajustou-se a um modelo geológico de dobra anticlinal; e a quinta foi usada apenas na representação de tal modelo geológico.

\section{Introdução}

Sem dúvidas, o método do raio é um dos métodos mais poderosos e amplamente usados na investigação de problemas de propagação da onda na exploração geofísica moderna. Aplicado na interpretação sísmica, na modelagem sísmica, na localização de pontos difratores, no cálculo do tempo de trânsito da onda sísmica, etc. Algumas idéias fundamentais da teoria do raio têm sido conhecidas na física ao longo do tempo. Provavelmente a primeira descrição do método do raio, como uma ferramenta de estudo de problemas de propagação da onda na eletrodinâmica, foi dada por R. K. Luneburg nos anos 40. A partir do final dos anos 50 , o método do raio começou sua trajetória triunfal na geofísica. Existe um certo consenso entre os cientistas de que o raio sísmico, enquanto ente físico, tem sentido apenas no domínio das altas freqüências, onde ele teria um caráter similar aos raios luminosos. Apesar do raio não gozar do mesmo status de existência física conferido à onda, nada nos impede de traçar linhas perpendiculares, no caso isotrópico, às frentes de ondas (isócronas). Tais linhas, vistas como raios, têm um caráter artificial, mas, sobre elas podemos calcular, dentre outras propriedades da onda, tempos de trânsito.

Obtenção de campos de tempos de trânsito em modelos de campos de velocidades sísmicas (que representam aspectos sísmicos de meios e modelos geológicos) são de grande utilidade em importantes domínios da sísmica tais como: migração (MRT: migração reversa no tempo), inversão (tomografia sísmica), sísmica teórica (resolução da equação de transporte), modelagem (geração de perfis de tempo de viagem em superfície e em poços) e etc. As equações das trajetórias dos raios sísmicos resultam do princípio de Fermat aplicado à função dada pela integral da vagarosidade calculada ao longo de caminhos que conectam dois pontos do campo de velocidades e que assume valores que são tempos de trânsito da onda em sua viagem entre os mencionados pontos (Popov, 2002). Isto é, a trajetória, $C$, de um raio originado num ponto $A$ e que chega num outro $B$, ambos pertencentes ao campo, é aquela que minimiza o funcional

$t(C)=\int \frac{d s}{V}$, onde $\mathrm{V}$ é a velocidade, ds é o elemento de comprímento de arco e t é o tempo de trânsito. Uma vez obtida a trajetória do raio, o tempo de trânsito é obtido ao longo dela. Resolver o problema de traçar raios conectando dois pontos de um modelo não é, em geral, uma tarefa trivial (o método paraxial é um exemplo de abordagem para a resolução deste problema; Figueiró \& Madariaga, 2000). Entretanto, O traçamento de raios em modelos com certo grau de complexidade é uma tarefa relativamente fácil quando comparada à simulação da propagação ondulatório nestes mesmos modelos, tendo, portanto, implicações no alto custo computacional da abordagem sísmica da onda comparativamente ao do raio.

\section{Metodologia/ Problema Investigado}

A base teórica da qual partimos para a realização do traçamento de raios (nos campos de velocidades acústicas, $\mathrm{V}(\mathrm{x}, \mathrm{z})$, que representam meios isotrópicos bidimensionais, onde $x$ representa uma posição horizontal na superfície de observação e z profundidade) são as equações do raio (Cervený, 1987) dada por:

$$
\frac{d \vec{X}(\tau)}{d \tau}=\vec{P}(\tau)(1.1) \text { e } \frac{d \vec{P}(\tau)}{d \tau}=\frac{1}{2} \vec{\nabla}\left[\frac{1}{V^{2}}\right]
$$

Onde $\vec{X}(\tau)=(x(\tau), z(\tau))$ é o vetor posição dos pontos da trajetória do raio; $\vec{P}(\tau)$ é o vetor tangente à trajetória do raio no ponto $(x(\tau), z(\tau))$ denominado vetor vagarosidade, $\mathrm{V}=\mathrm{V}(\mathrm{x}, \mathrm{z})$ é a velocidade de propagação da onda compressional no ponto $(x, z)$ do modelo sísmico 
adotado e $\tau$ é um parâmetro do caminho seguido pelo raio, definido por:

$$
\tau=\int_{0}^{t} V^{2} d t
$$

Com t sendo o tempo de trânsito medido ao longo do raio até o ponto $\vec{X}(\tau)$. Os raios são traçados utilizando-se expansões em série de Taylor de primeira ordem da trajetória do raio e do vetor vagarosidade com auxílio das equações do raio (1.1) e (1.2), tal como segue:

$$
\vec{X}(\delta \tau)=\vec{X}(0)+\frac{d \vec{X}(0)}{d \tau} \cdot \delta \tau=\vec{X}(0)+\vec{P}(0) \cdot \delta \tau
$$

e

$$
\vec{P}(\delta \tau)=\vec{P}(0)+\frac{d \vec{P}(0)}{d \tau} \cdot \delta \tau=\vec{P}(0)+\frac{1}{2} \vec{\nabla}\left[\left.\frac{1}{V^{2}(x, z)}\right|_{\tau=0}\right] \cdot \delta \tau
$$

Ao final de cada passo do processo de construção da trajetória do raio o vetor vagarosidade deve sofrer uma atualização adicional a fim de satisfazer a equação eiconal

$$
\|\vec{P}\|_{2}=\sqrt{P_{1}^{2}+P_{2}^{2}}=\frac{1}{V(x, z)},
$$

onde $\vec{P}=\left(P_{1}, P_{2}\right)$. Esta formulação contém todos os ingredientes necessários para a obtenção dos caminhos percorridos pelos raios, bem como um dos atributos do raio conhecido por tempo de trânsito ao longo dos raios. Como as equações do raio são claras e explicitamente formuladas, então a implementação em código computacional do traçado do raio se dá de modo simples e elegante.

Um método direto para calcular, numericamente, o tempo de trânsito é usar o próprio traçamento de raios no modelo. Isto é, o tempo em cada nó da poligonal que representa a trajetória é feito concomitantemente com o seu traçamento. Um feixe de raios origina-se de uma fonte na superfície e é traçado em subsuperfície. O raio sofre um encurvamento à medida que ele encontra variações na velocidade do modelo. O tempo gasto pelo raio para realizar a viagem ao longo da trajetória que conecta a posição da fonte, $\mathrm{S}$, àquela de um ponto $\left(\mathrm{X}_{\mathrm{N}+1}\right.$, $Z_{N+1}$ ) situado sobre esta mesma trajetória é calculado numericamente e é dado por:

$$
T\left(x_{N+1}, z_{N+1}\right)=\sum_{i=0}^{N} \frac{1}{V_{i}} \cdot \sqrt{\left(x_{i+1}-x_{i}\right)^{2}+\left(z_{i+1}-z_{i}\right)^{2}}
$$

Em termos de economia computacional, é interessante escrever (1.7) do seguinte modo:

$$
T\left(x_{N+1}, Z_{N+1}\right)=T\left(x_{N}, Z_{N}\right)+\frac{1}{V_{N}} \sqrt{\left(x_{N+1}-x_{N}\right)^{2}+\left(z_{N+1}-Z_{N}\right)^{2}}
$$

supondo que $\mathrm{T}\left(\mathrm{x}_{0}, \mathrm{Z}_{0}\right)=0$.
Um modelo sísmico é construído a partir de informações extraídas do modelo geológico. Tal modelo sísmico é, na verdade, um campo discretizado de velocidades sísmicas de ondas compressionais. Procura-se, então, ajustar um polinômio da forma

$$
V(x, z)=\sum_{i+j=0}^{N} c_{i, j} x^{i} z^{j} \cdot(1.9)
$$

Mas sinteticamente, a expressão (1.9) consiste em: $A C=V$, onde " $A$ " é a matriz que contém a forma polinomial, "C" é o vetor dos coeficientes e "V" é o vetor das velocidades nos nós discretizados. O método de mínimos quadrados dá a seguinte solução:

$$
C=\left(A^{T} A\right)^{-1} \cdot A^{T} \cdot V
$$

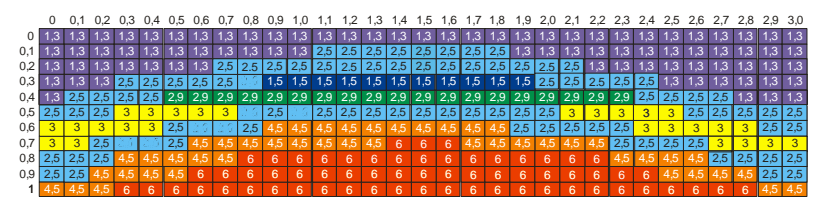

Figura1- Modelo geológico de uma dobra anticlinal discretizado

\section{Resultados}

\section{$M_{1}$ : Modelo com variação linear lateral e vertical}

A Figura2 mostra um campo de velocidades genérico que pode ser interpretado como ambiente de camadas sedimentares planas e paralelas que sofreu uma rotação que resultou na produção de um sistema de camadas inclinadas.

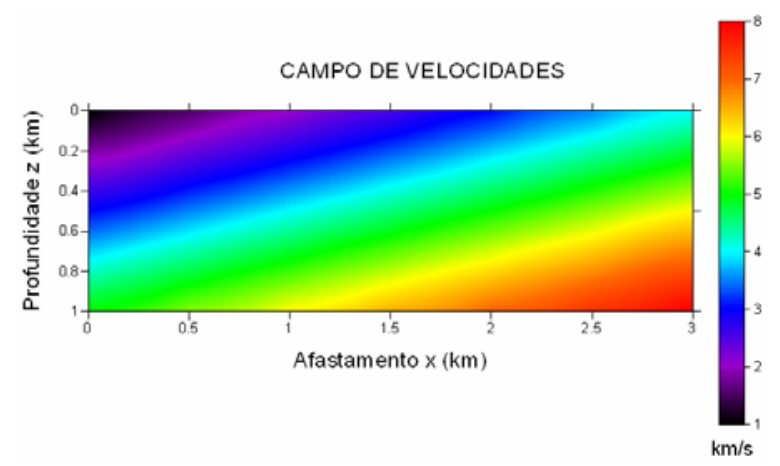

Figura2- campo de velocidades representado pela função $v_{1}=1,0+1,0 . x+4,0 . z$

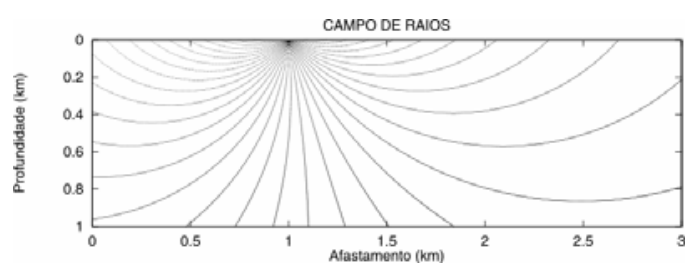


Figura3- Campo de raios para o campo velocidades representado por $v_{1}$

A Figura3 mostra o raio, que parte da fonte numa direção quase perpendicular às interfaces das camadas, tende a ter uma trajetória retilínea, enquanto os outros se encurvam mais facilmente.

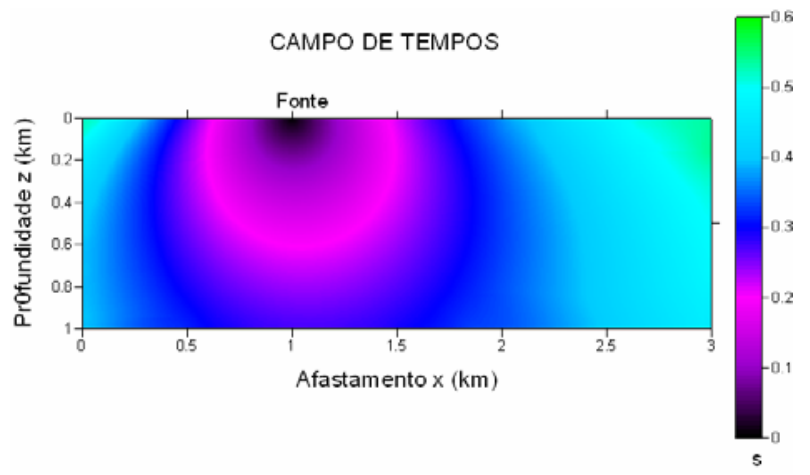

Figura4- Campo de tempos para o campo velocidades representado por $v_{1}$

$\mathrm{Na}$ Figura 4 as isócronas tendem apresentar maior deformação para direita e para baixo, pois elas tendem a se alongar mais nas regiões de velocidades mais altas.

\section{$M_{\| l}$ : Modelo com variação lateral e vertical quadrática}

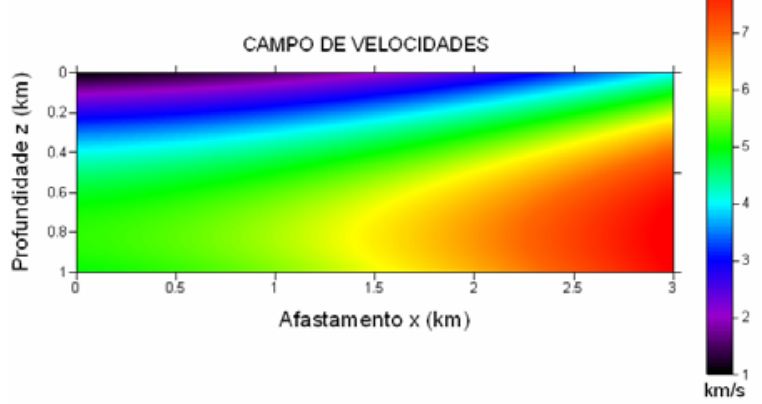

Figura5- campo de velocidades representado pela função $v_{2}(x, z)=1,0+0,25 \cdot x+10,0 . z+0,25 \cdot x^{2}-6,0 . z^{2}$

Na Figura 5 mostra um campo genérico de velocidades com uma região de altas velocidades no lado direito inferior que pode ser interpretado geologicamente como uma intrusão magmática responsável pela compressão das camadas no lado direito do modelo .

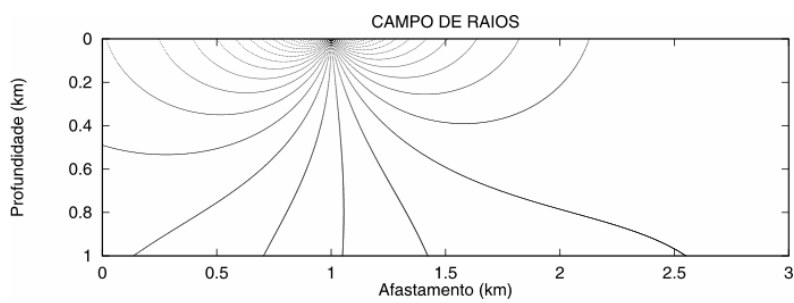

Figura6- Campo de raios para o campo velocidades representado por $v_{2}$

Os raios da Figura 6 mudam de direção ao encontrarem uma variação de velocidades, principalmente no lado direito inferior da figura. Uma aquisição sísmica com as configurações abaixo iria obter poucas informações desta região.

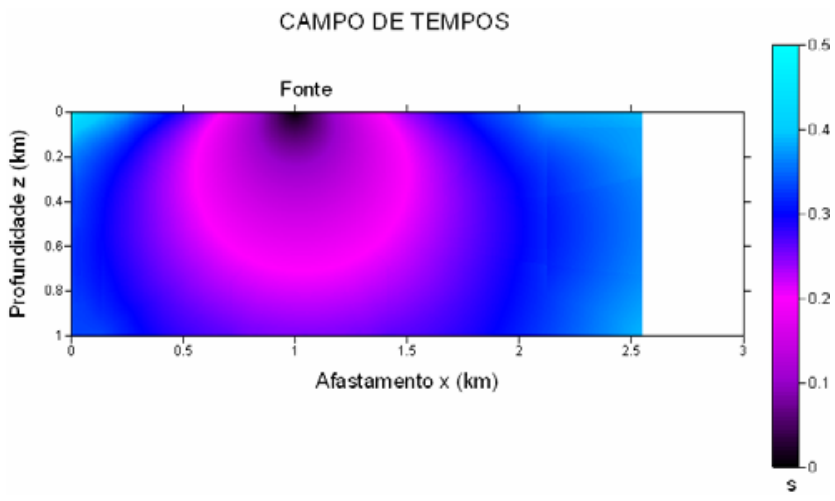

Figura7- Campo de tempos para o campo de velocidades representado por $v_{2}$.

A Figura 6 mostrou que os raios não percorreram a região destra da figura, com isso não conseguimos obter tempos de trânsito dos raios sísmicos nesta região.

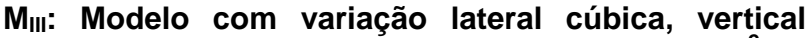
quadrática, com termos cruzados do tipo $x z$ e $x^{2} z$ e ajustado ao modelo geológico da dobra anticlinal.

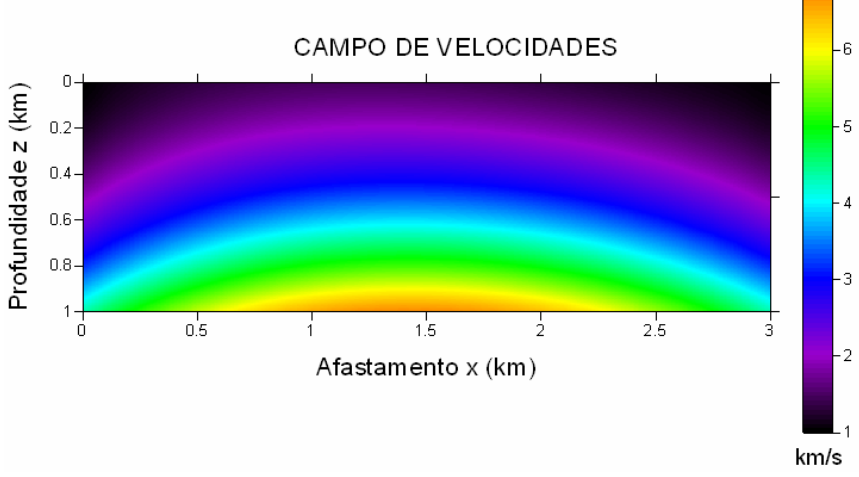

Figura8- Campo de velocidades ajustado ao modelo geológico de uma dobra anticlinal representado pela função

$v_{4-B}(x, z)=1,0+0,8622 \cdot x+0,2834 \cdot z-0,4694 \cdot x^{2}+3,0301 \cdot z^{2}+2,6483 \cdot x z+0,0613 \cdot x^{3}-0,8846 \cdot x^{2} z$

Os Raios da Figura 9 obedecem à lei de Snell, ou seja, os raios se afastam da normal à medida que passa de um meio de baixa velocidade para outro com velocidade maior. 


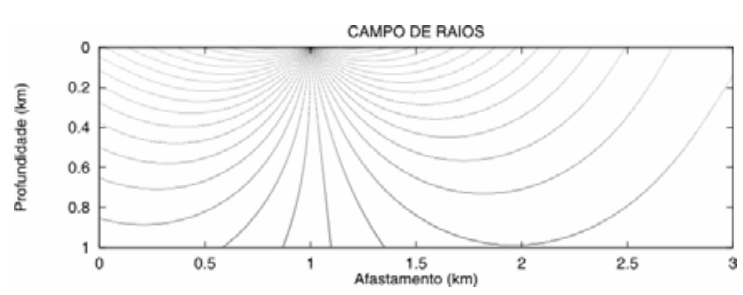

Figura9- Campo de raios para o campo de velocidades $v_{4-b .}$

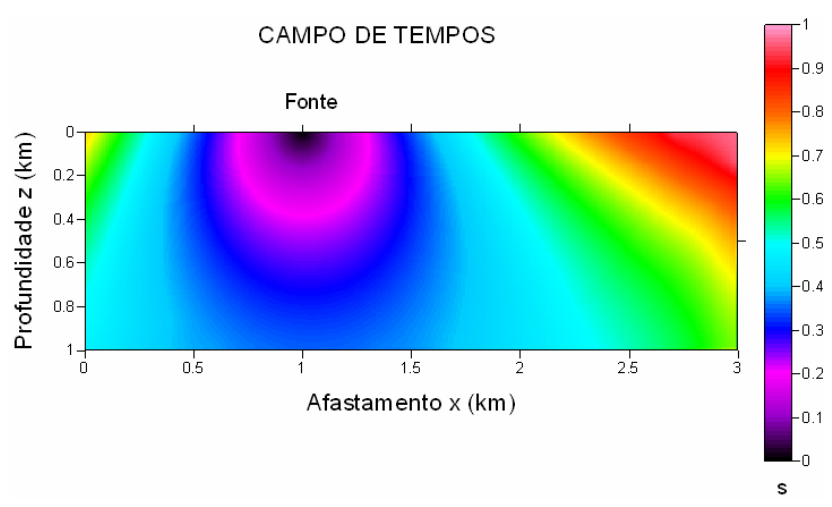

Figura10- Campo de tempos para o campo de velocidades $v_{4-b .}$

$M_{\mathrm{IV}}$ : Modelo com variação lateral e vertical cúbicas, com termos cruzados do tipo $x z, x^{2} z$ e $x z^{2}$, ajustado ao modelo geológico de dobra anticlinal.

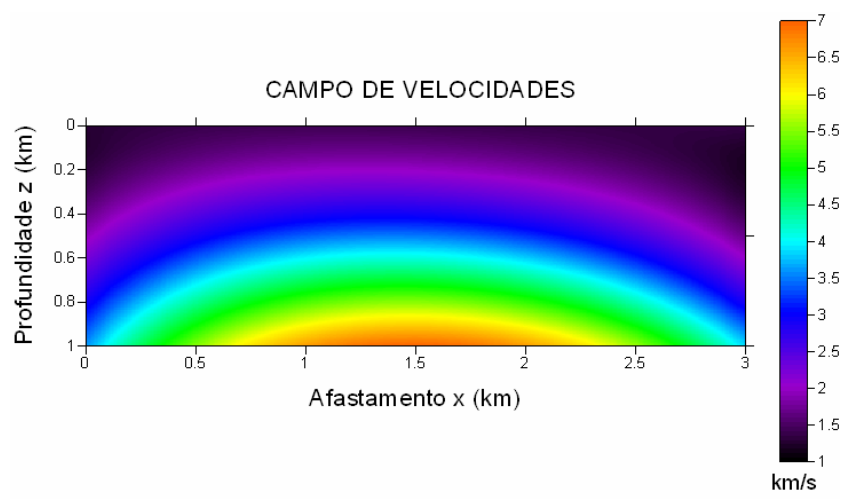

Figura11- Campo de velocidades ajustado ao modelo geológico de uma dobra anticlinal representado pela função

$v_{5-B}(x, z)=1,2528+0,3652 \cdot x-0,5339 \cdot z-0,2436 \cdot x^{2}+4,6729 . z^{2}$

$+3,6842 \cdot x z+0,0447 \cdot x^{3}-1,4034 \cdot x^{2} z+0,5836 \cdot x z^{2}-1,812 \cdot z^{3}$

Este campo de velocidades também foi ajustado pelo método dos mínimos quadrados, mas este conseguiu aproximar-se um pouco mais do modelo geológico proposto, pois adicionamos dois novos termos ao polinômio.

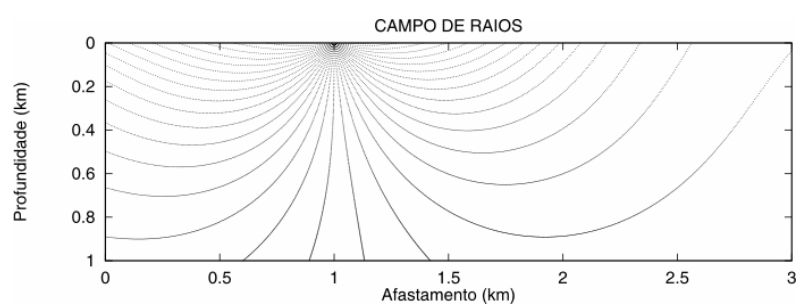

Figura12- Campo de raios para o campo de velocidades $v_{5-b .}$

A Figura13 mostra que para a fonte localizada em $x=1,0$ $\mathrm{km}$, as isócronas tendem a apresentar maior deformação para direita, pois os raios sofrem maior influência da anticlinal, maior velocidade. Observar que o domo apresenta sua maior extensão vertical em $x=1,5 \mathrm{~km}$.

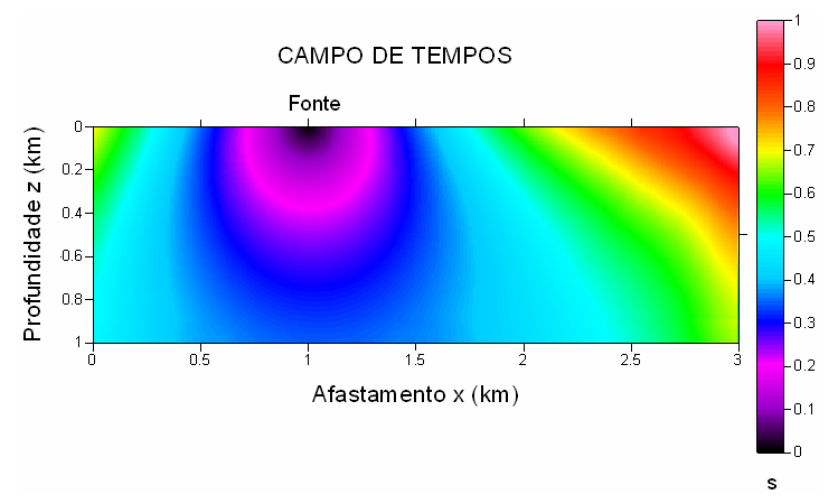

Figura13- Campo de tempos para o campo de velocidades $v_{5-b}$

\section{Discussão e Conclusões}

Este trabalho mostrou que através do traçamento de raios podemos também obter campos de tempos em campos de velocidades com parametrização polinomial. Os resultados se apresentaram de forma satisfatória, tendo em vista que as trajetórias dos raios se encurvaram à medida que o raio encontrou variação de velocidade no modelo, obedecendo a lei física, como lei de Snell, princípio de Fermat e o princípio da reversibilidade. Os campos de tempos apresentaram-se como esperado, ou seja, o raio gastou, para percorrer a mesma distância, menos tempo em regiões de altas velocidades do que naquelas de menores, observar a deformação das isócronas. Observamos que o método do raio é um método de interpretação e modelagem muito rápido. Até um certo nível, quanto mais refinada for a discretização, melhor será a representação do modelo geológico pelo polinômio, entretanto chega-se a um ponto em que não adianta refinar a discretização, pois a amarração polinomial impede uma melhora. 


\section{Agradecimentos}

O autor agradece aos seus pais, Ruy e Lúcia, e seus irmãos , Romel e Rafael, pelo amor e pela paciência. Aos professores do CPGG-UFBA, em especial ao seu orientador, Wilson Figueiró, pelos conhecimentos. À ANP pela oportunidade de trabalho e pelo suporte financeiro.

\section{Referências}

Cervený, V., 1987. Ray method for Three-Dimensional Seismic Modeling. Petroleum Industry Course, the Norwegian Institute of Techonology.

Figueiró, W. \& M.,Madariaga, R. I.,2000. Three dimensional two-points paraxial ray tracing problem in presence of caustics. $6^{\text {th }}$ International Congress of Brazilian Geophysical Society. CDROOM, Rio99, Rio de Janeiro.

Menke,W., 1989. Geophysical data analysis: Discrete inverse theory. International geophysics series, Academic Press, Volume 45.

Popov, M. M., 2002. Ray theory and Gaussian beam method for geophysicists. Editora da Universidade Federal da Bahia, Salvador, Bahia. 